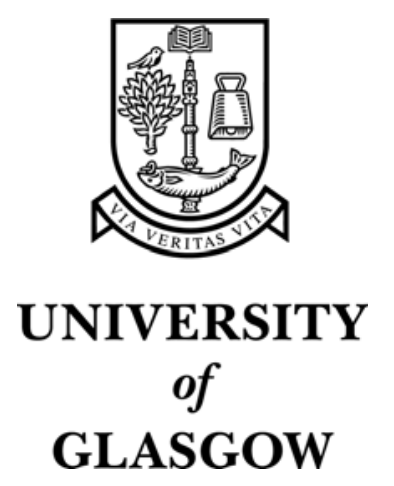

Miller, T.J.E. (1995) Faults and unbalance forces in the switched reluctance machine. IEEE Transactions on Industry Applications 31(2):pp. 319-328.

http://eprints.gla.ac.uk/archive/00002843/ 


\title{
Faults and Unbalance Forces in the Switched Reluctance Machine
}

\author{
Timothy J. E. Miller, Senior Member, IEEE
}

\begin{abstract}
The paper identifies and analyzes a number of severe fault conditions that can occur in the switched reluctance machine, from the electrical and mechanical points of view. It is shown how the currents, torques, and forces may be estimated, and examples are included showing the possibility of large lateral forces on the rotor. The methods used for analysis include finiteelement analysis, magnetic circuit models, and experiments on a small machine specially modified for the measurement of forces and magnetization characteristics when the rotor is off-center. Also described is a computer program (PC-SRD Dynamic) which is used for simulating operation under fault conditions as well as normal conditions. The paper discusses various electrical configurations of windings and controller circuits, along with methods of fault detection and protective relaying. The paper attempts to cover several analytical and experimental aspects as well as methods of detection and protection.
\end{abstract}

\section{NOMENCLATURE}

Symbols
$F$
$F$
$F$
$h$
Overlap
$r$
$R$
$T$
$\Phi$
$\theta$

Subscripts

1,2 . . total

$x, y$

$\begin{array}{ll}\begin{array}{l}\text { Magnetomotive } \\ \text { force } \\ \text { (MMF) }\end{array} & \text { Ampere-turns (At). } \\ \begin{array}{l}\text { Force } \\ \text { Rotor offset }\end{array} & \mathrm{N} . \\ \begin{array}{l}\text { Overlap angle } \\ \text { Rotor radius }\end{array} & \text { degrees. } \\ \text { Reluctance } & \mathrm{m} . \\ \text { Torque } & \mathrm{At} / \mathrm{Wb} . \\ \text { Flux } & \mathrm{Nm} \\ \text { Rotor position } & \mathrm{Wb} . \\ & \text { radians or degrees. }\end{array}$

fault conditions of extreme severity can occur. The paper concentrates on unbalanced faults, particularly those that can produce unbalanced magnetic side-pull on the rotor. It is shown that these forces are periodic, highly concentrated, and potentially very large. The analysis features simple analytical methods as well as detailed finite-element analysis, and it is shown how transients leading to the generation of unbalanced forces are simulated using the PC-SRD Dynamic computer program. The analysis is backed up by measurements on a specially modified machine. The paper also discusses possible winding arrangements and protection schemes and tries to give a comprehensive view of the problem and of methods for dealing with it.

\section{ElECTRICAL UNBALANCE}

\section{A. Effects of an Open-Circuit Fault}

The phase windings of SR machines are often shown with two opposite pole-coils ( 1 and 4 in Fig. 1). Normally these pole-coils have the same ampere-turns $F$, producing a balanced flux $\Phi$ that crosses the rotor with perfect symmetry. Using the pole numbers defined in Fig. 1, this symmetry is expressed by $F_{1}=F_{4}$ and $\Phi_{1}=\Phi_{4}$. The fluxes in unexcited poles $2,3,5$, and 6 are zero and the magnetic forces of attraction are exactly balanced.

When one of the pole-coils is open-circuited, the flux becomes unbalanced as shown in Fig. 1. Fluxes appear in the adjacent, unexcited poles. As the rotor rotates, the flux pattern changes in a complex manner. It will be shown that the main source of unbalance force is the difference in magnetic pull between opposite poles (i.e., 1 and 4 in Fig. 1). Typically, the peak flux-density in the healthy pole may be twice that in the open-circuited pole, giving rise to a $4: 1$ ratio of the magnetic pulls. This can be inferred from Fig. 2, which plots the radial flux-density around the airgap under poles 1 and 4, showing nearly a $2: 1$ ratio in the densities.

\section{INTRODUCTION}

$\mathbf{T}$ HE switched reluctance (SR) machine is being researched for aerospace and industrial applications including motor drives, actuators, and starter-generators. Although it has been widely acclaimed as "fault tolerant" [1], [2], certain

Paper IPCSD 94-62, approved by the Electric Machines Committee of the IEEE Industry Applications Society for presentation at the 1993 Industry Applications Society Annual Meeting, Toronto, Ontario, Canada, October 3-8. Manuscript released for publication August 12, 1994.

The author is with SPEED Laboratory, University of Glasgow, Glasgow, G12 8LT, Scotland, UK.

IEEE Log Number 9407573.

\section{B. Waveform and Magnitude of the Lateral Force}

The waveform of the lateral force as the rotor rotates is clearly the result of complex changes in the flux pattern, and also depends on the currents in the coils. To begin with, a simplified magnetic-circuit analysis is used to determine the general behavior. Then a detailed analysis is performed using finite-elements. Finally, the analysis is calibrated by measurement on a specially modified machine.

To estimate how the lateral force varies as the rotor rotates, the magnetic circuit is modeled by a reluctance network as 


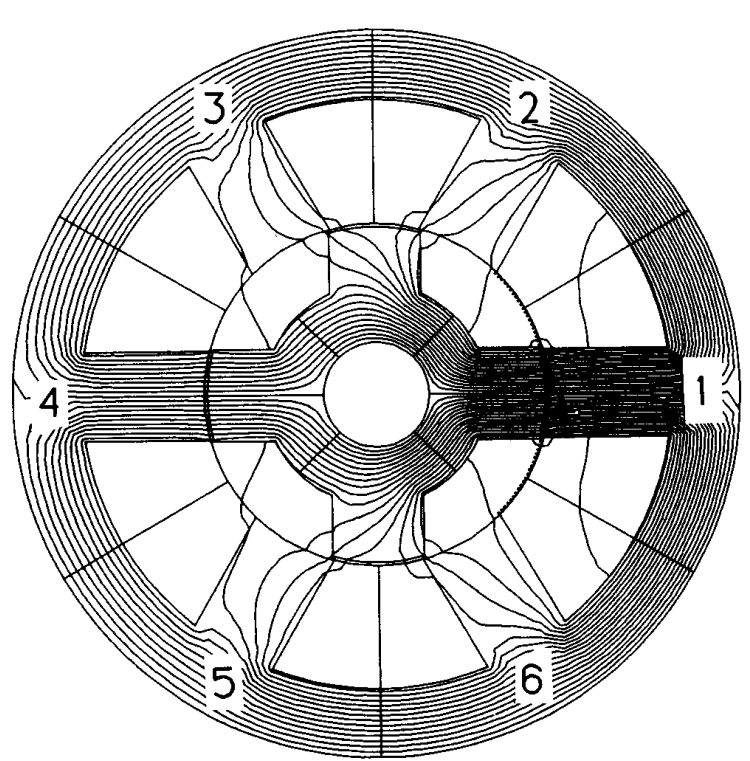

Fig. 1. Finite-element flux-plot of switched reluctance motor with excitation on pole 1 only. (Pole-coil 4 is open-circuited.) The pole numbering scheme shown here is used throughout the paper.

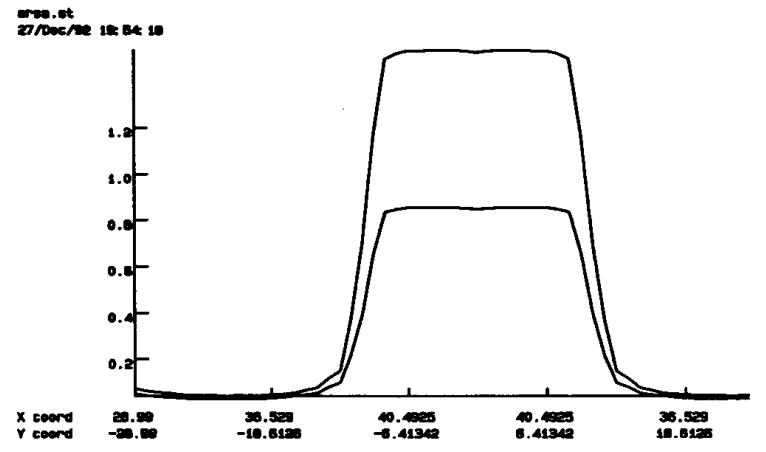

Fig. 2. Flux-density distribution under poles 1 and 4 , corresponding to the unbalanced excitation in Fig. 1.

shown in Fig. 3. The reluctances of the stator and rotor yokes are assumed to be negligible compared to the reluctances between overlapping poles. Each reluctance in Fig. 3 represents an irregularly shaped gap between the stator and the rotor. Of course, the reluctances are magnetically nonlinear and they are functions of the amount of overlap between the relevant poles.

It is necessary to determine how the flux divides among the six branches of Fig. 3 at all rotor positions. The rotor starts at the unaligned position (defined as $45^{\circ}$ ), and proceeds to the aligned position (defined as $90^{\circ}$ ). At that position the current is switched off. If we assume constant current in pole-coil 1 (corresponding to low-speed motoring operation), we can successively analyze the magnetic field and forces at successive positions. The analysis is performed by means of a spreadsheet program in tabular form. Seven columns represent seven rotor positions between the unaligned and aligned positions, with a step of $7.5^{\circ}$. The analysis begins with the amount of overlap between each stator pole and the

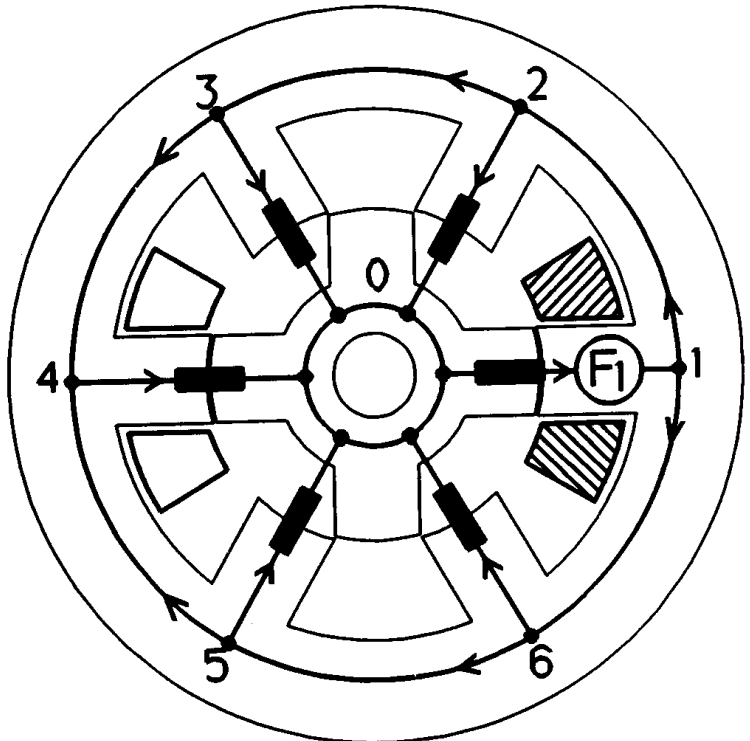

Fig. 3. Simple magnetic reluctance network.

corresponding rotor pole, at each rotor position, normalized to full overlap $\left(30^{\circ}\right)$. For example, at the aligned position the overlap under poles 1 and 4 is $30^{\circ}$ (1.00), but the overlap under all the other stator poles is zero. At the $82.5^{\circ}$ position, the overlap under poles 1 and 4 has decreased to $22.5^{\circ}$, while the overlap under poles 2 and 5 has grown to $7.5^{\circ}$. Under poles 3 and 6 it remains zero.

As the rotor rotates, the changing overlap angles modify the reluctances. Obviously the reluctance is at a minimum when the overlap is $30^{\circ}$. When the overlap is zero, the reluctance is of the order of four times the minimum reluctance, this being the value required to produce the $50 \%$ value of flux-density in pole 4 in Fig. 2. For example, at the aligned position with respect to poles 1 and 4 , the reluctances $R_{2}, R_{3}, R_{5}$, and $R_{6}$ are all 4.00 , while $R_{1}$ and $R_{4}$ are 1.00 .

To a first approximation, the per-unit reluctance variation with overlap-angle can be written

$$
R=\frac{1}{\frac{1}{4}+\frac{\text { Overlap }}{30} \times \frac{3}{4}} .
$$

At each rotor position, the network of Fig. 3 is solved, assuming constant MMF $F_{1}$ on pole 1 . The total reluctance "seen" by the MMF generator $F_{1}$ is given by

$$
R_{\text {total }}=R_{1}+\frac{1}{\frac{1}{R_{2}}+\cdots+\frac{1}{R_{6}}}
$$

and this determines the flux $\Phi_{1}$ in pole 1 as $F_{1} / R_{\text {total }}$. The fluxes in all the other stator/rotor reluctances are then calculated from the network according to their respective values. For example,

$$
\Phi_{2}=\Phi_{1} \times \frac{R_{\text {total }}}{R_{2}} .
$$

The flux-densities within each overlap area are estimated by dividing each flux by the corresponding per-unit overlap 


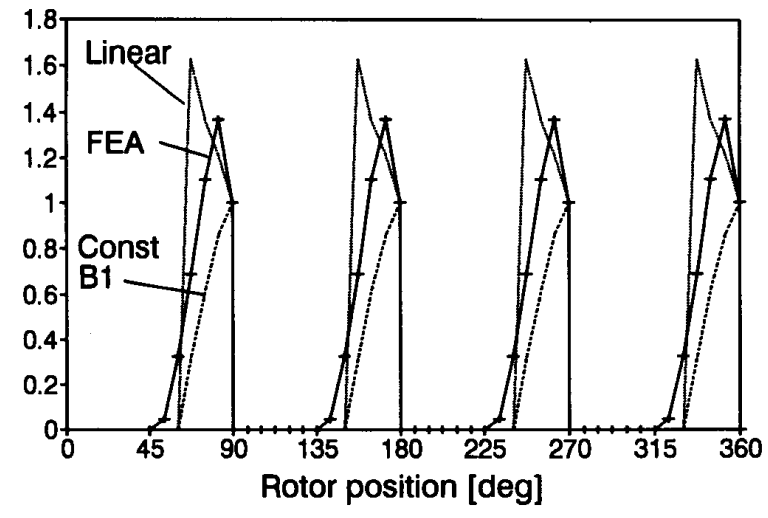

Fig. 4. Lateral force versus rotor position. Linear-Calculated by means of the reluctance network in Fig. 3, assuming infinitely-permeable steel. Const. B $1-$ Calculated by means of the reluctance network in Fig. 3, assuming constant flux-density in the overlap region between stator and rotor poles. FEA - Calculated by the finite-element method with Maxwell stress.

angle. The forces across each stator/rotor gap are calculated from the square of the flux-density times the overlap angle. They are assumed to act radially along the center-lines of the stator poles, and are resolved into $x$ - and $y$-components and summed to produce totals $F_{x}$ and $F_{y}$, normalized to the net force in the aligned position.

Fig. 4 shows the variation of $F_{x}$ over one revolution. The "linear" graph in Fig. 4 is calculated assuming infintely permeable steel. The force does not materialize until there is some overlap $15^{\circ}$ after the unaligned position (i.e., at $60^{\circ}$ ). It rapidly builds to a maximum at $67.5^{\circ}$. In this as in all positions, the $x$-forces under poles 2 and 5 cancel, and the resultant is always due to the difference in magnetic pull between poles 1 and 4. $F_{x}$ disappears at the aligned position because the current is switched off at that point.

The peak $F_{x}$ occurs at a partial-overlap position and appears to be 1.6 times the value at the aligned position, but this may be pessimistic because saturation limits the flux-density as the overlap decreases. To take this into account, the model is modified by assuming that pole 1 acts as a flux source whose normalized value is equal to the per-unit overlap $\theta_{1}$ under pole 1. This is equivalent to assuming that all the MMF is taken up in saturating pole 1 to a fixed flux-density in the region of overlap. Then the remaining poles are in parallel and their fluxes are calculated as before, e.g., in pole 2

$$
\Phi_{2}=\Phi_{1} \times \frac{R_{\text {total }}}{R_{2}} .
$$

The remaining procedure is the same as before, and the result is the graph labelled "Const. B1" in Fig. 4.

The two extreme cases (linear and saturated) suggest that saturation decreases the rate of rise of the unbalance force, and it appears to limit the peak value. The result of a finite-element investigation is also shown in Fig. 4, and Fig. 5 shows a typical flux-plot. The finite-element graph (labelled "FEA") is intermediate between the "linear" and saturated graphs in Fig. 4 , as would be expected since the two simplified calculations represent extreme limiting cases.

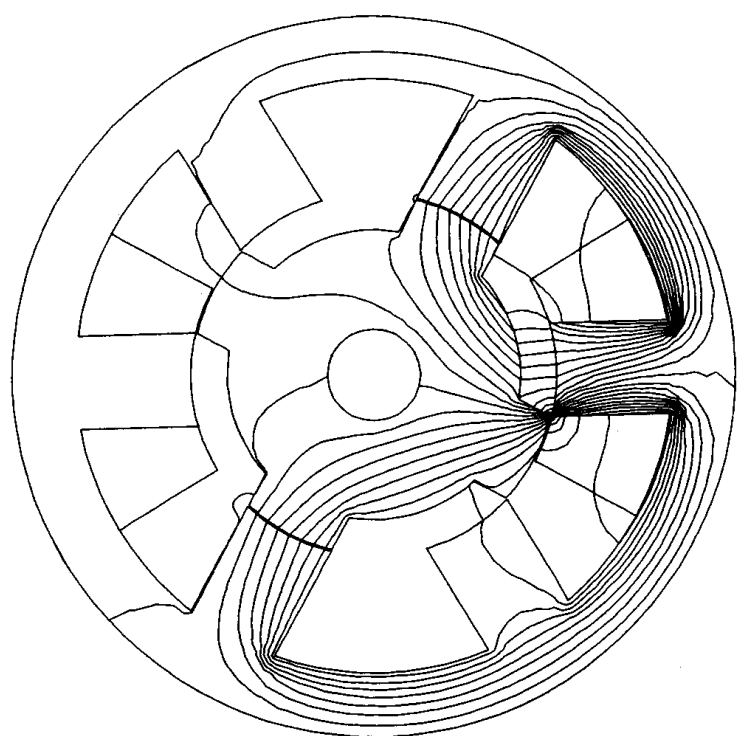

Fig. 5. Flux plot at a typical intermediate position $\left(60^{\circ}\right)$ between unaligned $\left(45^{\circ}\right)$ and aligned $\left(90^{\circ}\right)$.

\section{Scale of the Force}

The finite-element force calculation integrates Maxwell's stress over the rotor surface, so it should be possible to calculate the force in the aligned position (see Fig. 1) within a few percent of the finite-element value, using the formula $B^{2} / 2 \mu_{0}$ for the force per unit area between two magnetized surfaces. The maximum (saturation) value of $B$ ordinarily does not exceed about $1.7 \mathrm{~T}$ in silicon steel or $2.1 \mathrm{~T}$ in cobaltiron, giving values of about $170 \mathrm{psi}$ and $250 \mathrm{psi}\left(1.2 \times 10^{5}\right.$ $\mathrm{kgf} / \mathrm{m}^{2}$ and $1.8 \times 10^{5} \mathrm{kgf} / \mathrm{m}^{2}$ ) respectively. With one coil opencircuited, the net force will have a peak value of the order of $250 \times(1-1 / 4)=190 \mathrm{psi}\left(1.3 \times 10^{5} \mathrm{kgf} / \mathrm{m}^{2}\right)$. For example, in a machine with a 4 -in $(100 \mathrm{~mm})$ rotor diameter, $30^{\circ}$ pole arcs, and 4-in $(100 \mathrm{~mm})$ stack length, the lateral force could peak at $800 \mathrm{lbf}(363 \mathrm{kgf})$.

\section{Effect of a Short-Circuit Fault}

When one pole-coil is short-circuited, even with one shorted turn, induced currents keep its flux-linkage nearly zero while the flux builds up in the healthy pole. In effect $R_{4}$ is dynamically infinite, and $R_{\text {total }}$ will be dynamically increased. The effect on the forces can be estimated with the help of (1) and (2). In the aligned position, using normalized values, $R_{1}=1$ and $R_{2}=R_{3}=R_{5}=R_{6}=4$. For the faulted pole, $R_{4}=1$ if pole-coil 4 is open-circuited, or 0 if pole-coil 4 is shortcircuited. Accordingly, when pole-coil 4 is open-circuited we have

$$
R_{\text {total }}=1+\frac{1}{\frac{1}{4}+\frac{1}{4}+1+\frac{1}{4}+\frac{1}{4}}=\frac{3}{2} .
$$

When pole-coil 4 is short-circuited we have

$$
R_{\text {total }}=1+\frac{1}{\frac{1}{4}+\frac{1}{4}+0+\frac{1}{4}+\frac{1}{4}}=2 .
$$




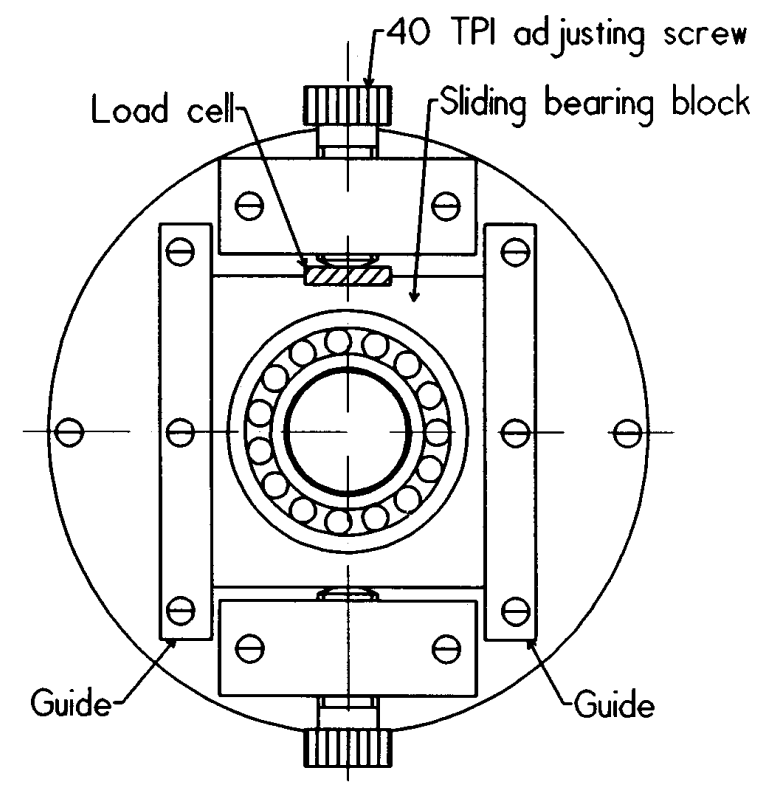

Fig. 6. Sliding bearing block with load cell for lateral force measurement.

The flux $\Phi_{1}$ in pole 1 is reduced by the inverse ratio of $R_{\text {total }}$, i.e., to $(3 / 2) / 2=3 / 4$ or $75 \%$ of the value in the opencircuit case. If we assume that the peak lateral force at the aligned position is proportional to $\Phi_{1}^{2}-\Phi_{4}^{2}$, then the ratio of this force in the two cases is $\left[1^{2}-(1 / 2)^{2}\right] /\left[(3 / 4)^{2}-0^{2}\right]=4 / 3$ : i.e., the short circuit on pole-coil 4 increases the lateral unbalance force by $33 \%$. The flux is diverted into poles 2 , 3,5 , and 6 but because of the balancing effect of symmetry, the $F_{y}$ should remain zero or relatively small.

\section{E. Measurement of the Force}

A small machine has been specially adapted to permit controlled eccentric deflection of the rotor and the measurement of the unbalanced forces. A simplified drawing of this machine is shown in Fig. 6. As shown, the apparatus measures force in the vertically upward direction. The whole assembly (one at each end of the machine) can be rotated relative to the stator frame. Force can be measured with the rotor either centered or with its axis displaced in any direction.

Fig. 7 shows typical measurement of the lateral force in the aligned position with one pole-coil (1) excited and the opposite pole-coil (4) open-circuited. The force is plotted as a function of the current in pole-coil 1 . The motor is a $6 / 4$ motor with a stack length of $50 \mathrm{~mm}$, pole width $10 \mathrm{~mm}$, normal airgap length $0.25 \mathrm{~mm}$, and $150 \mathrm{turns} /$ pole. This data is sufficient to make rough checks on the validity of the experimental measurements, but it is left for a future paper to present a detailed theoretical treatment in which the measured forces are compared with finite-element calculations.

With the rotor in the centered position, the force versus current curve has the expected $S$-shaped characteristic attributable to a $B^{2}$ variation of force at lower currents, with a levelling off due to saturation at higher currents.

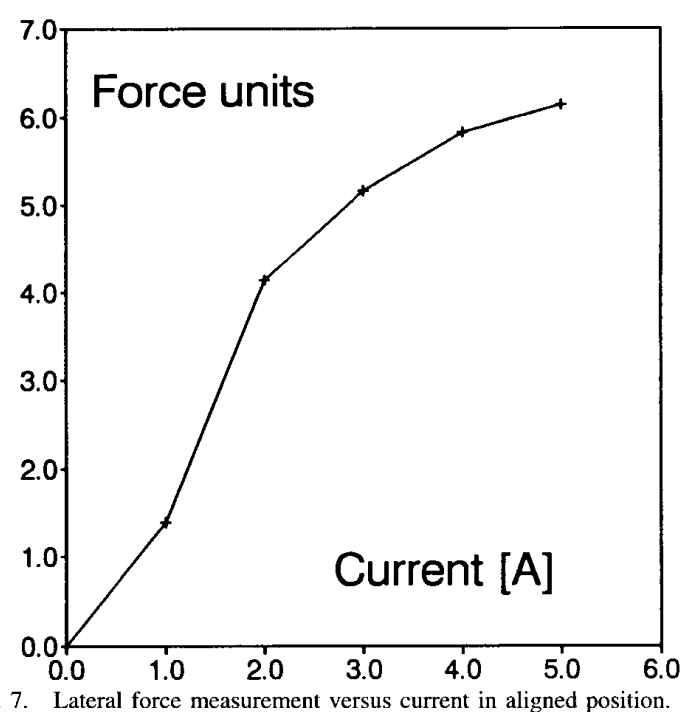

When the rotor is displaced downwards by means of the adjusting screws (see Fig. 6), it moves closer to the healthy pole and further from the faulty pole. Consequently the airgap under the healthy pole is decreased. The force curve now saturates at a lower current level. The saturated value is slightly reduced, possibly because the fringing field at the sides of the poles is reduced when the airgap is closed up. When the rotor is moved the other way, i.e., upwards and away from the healthy pole, the airgap under this pole increases and saturation does not appear until the current is much higher. Further analysis of the offset rotor is attempted in the next section.

\section{Mechanical UnBalance}

Mechanical unbalance can arise when the rotor is not properly centered in the stator, giving rise to different airgap lengths on opposite sides of the machine.

With equal MMF's in the opposite pole-coils, this mechanical offset would not cause a large offset force but for the fact that the force of attraction between rotor and stator poles has a very steep wavefront at the point where a rotor pole just begins to overlap a stator pole. Consider the production of torque by the azimuthal forces $F_{1}$ and $F_{2}$ in Fig. 8. Normally $F_{1}$ and $F_{2}$ are balanced: $F_{1}=F_{2}=F$. When the rotor is off-center, the forces may still have similar magnitudes but their waveforms may acquire a relative phase shift as shown in Fig. 9.

Since the torque is normally $T=2 F r$, we can infer that

$$
\Delta F=F_{1}-F_{2}=\frac{1}{2 r}\left[\frac{d T}{d \theta} \Delta \theta\right]
$$

where $d T / d \theta$ is the slope of the torque waveform normally produced by the two forces. If $h$ is the actual offset of the rotor, then $\Delta \theta \approx h / r$. Also $d T / d \theta$ can be estimated from the transient simulation carried out by PC-SRD Dynamic (see below). Hence the centering force can be estimated as

$$
\Delta F=\frac{h}{2 r^{2}} \frac{d T}{d \theta} \quad[\mathrm{N}]
$$

where $\theta$ is in radians and $h$ and $r$ are in meters. 


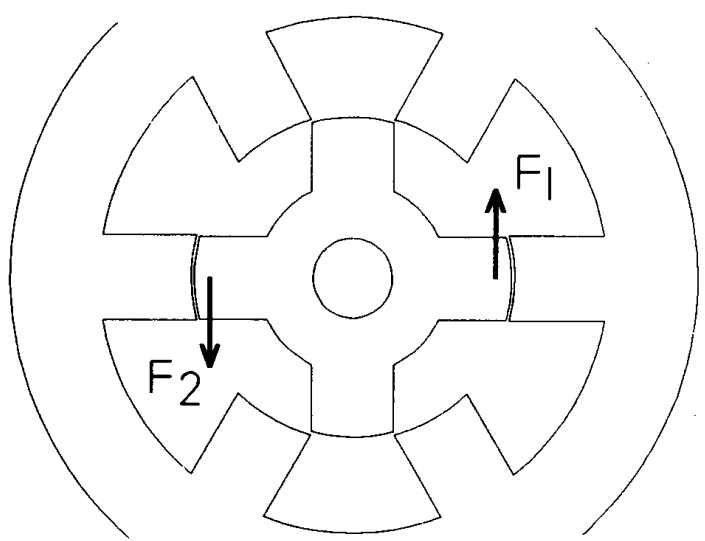

Fig. 8. Force production with offset rotor.

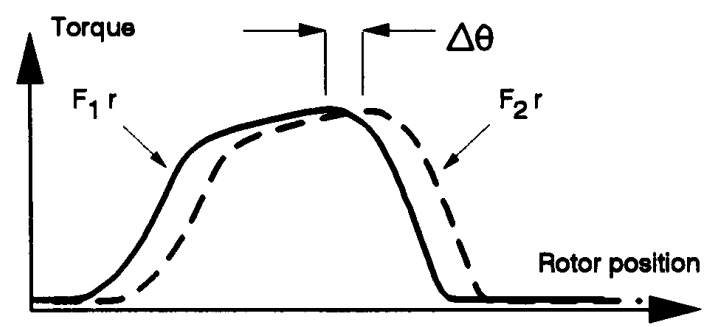

Fig. 9. Phase shift between azimuthal force waveforms.

This formula predicts fairly low levels of centering force due to mechanical offset. In a trial calculation for a machine with a rotor diameter of approximately 3.5 in and a stack length of 2 in, PC-SRD Dynamic predicted a torque waveform with a rising slope of $143 \mathrm{Nm} / \mathrm{rad}$ over approximately $25^{\circ}$ of rotation, and a falling slope of about $1700 \mathrm{Nm} / \mathrm{rad}$ over approximately $2^{\circ}$ of rotation. The rising slope produces a force of $24 \mathrm{~N}$ per $\mathrm{mm}$ of offset over $25^{\circ}$, and the falling slope produces about $310 \mathrm{~N}$ per $\mathrm{mm}$ of offset over $2^{\circ}$. Since the actual offset is likely to be much less than $1 \mathrm{~mm}$, the indication is that the forces are small. However, further experimental investigation is desirable to verify or disprove this.

\section{Simulation of TRANSIENTS}

In the extensive literature on the SR machine there are relatively few publications concerned with dynamics and control: Most authors concentrate on steady-state performance and motor design, and most of the control papers deal only with basic speed control (e.g., [6]). In this paper the $P C-S R D$ Dynamic computer program [1], [3]-[5] is used to simulate certain fault conditions and an example is shown in which the simulation provides necessary data for the calculation of the unbalanced forces discussed earlier.

\section{A. Description of the Program}

$P C-S R D$ Dynamic is well known as a CAD program for designing SR motors and their controllers, and earlier publications have focused on electromagnetic algorithms [3] and the method of simulation at fixed speed [4], [5]. The Dynamic version of $P C$-SRD models the complete control system including PID speed control, an analog/digital encoder model, variable firing angles, and inner-loop control by current regulation or voltage PWM. The structure of the simulation is shown in Fig. 10 , and includes five main elements:

1) An electromagnetic model of the SR motor and its power electronic controller;

2) A load model including load torque as functions of speed, rotor position, and step changes at predefined instants;

3) A PID velocity feedback loop with anti-windup and optional state feedback;

4) A firing-angle calculator that varies the firing angles with speed (in current- or voltage-regulation mode) or with speed error (in single-pulse mode); and

5) A model of the shaft position encoder with variable resolution and averaging algorithm for speed, including sample-and-hold delays.

Up to five phases may be modelled in detail over several revolutions, with a wide range of possible transient conditions initiated by changes in control variables or load changes. In addition, the program can simulate the effect of open- and short-circuit faults on any phase, as well as the effects of disturbances to the control system.

The program combines many of the features of finiteelement analysis and system simulation software in a single self-contained package, complete with its own editors and graphics. It incorporates many highly specialized algorithms that are peculiar to the SRM [3]-[5]. Some of these are very difficult to employ in connection with general-purpose simulation packages or finite-element programs, and even when they are so incorporated, the resulting process of simulation and analysis is slow and cumbersome.

\section{B. Response to a Short-Circuit Phase Fault}

Fig. 11 shows a transient simulation of an SR machine accelerating from $1000 \mathrm{r} / \mathrm{min}$ towards a reference speed of 4000 $\mathrm{r} / \mathrm{min}$ under PI control. After approximately $0.04 \mathrm{~s}$, a shortcircuit fault occurs on phase 1 . The loss of accelerating torque from phase 1 is evident in the average rate of acceleration, which falls to approximately $50 \%$ as the motor continues to accelerate with the other two phases. This ability to continue operating (albeit at a reduced output level), with the healthy phases unaffected by the faulted phase, is the reason why the SRM is claimed to be "fault tolerant."

Fig. 12 shows the current in phase 1 . Before the fault, the current is regulated normally to a peak value of $20 \mathrm{~A}$. The fault occurs during a period of diode conduction. The short-circuit isolates the phase winding from the demagnetizing voltage of the dc link (which is normally reverse-connected by the conducting freewheel diodes). Consequently the flux is not extinguished, and as soon as the aligned position is passed, phase 1 starts generating and the current is rapidly pumped up to $55 \mathrm{~A}$, nearly three times the regulated level. There follows an uncontrollable series of very large current pulses, during 


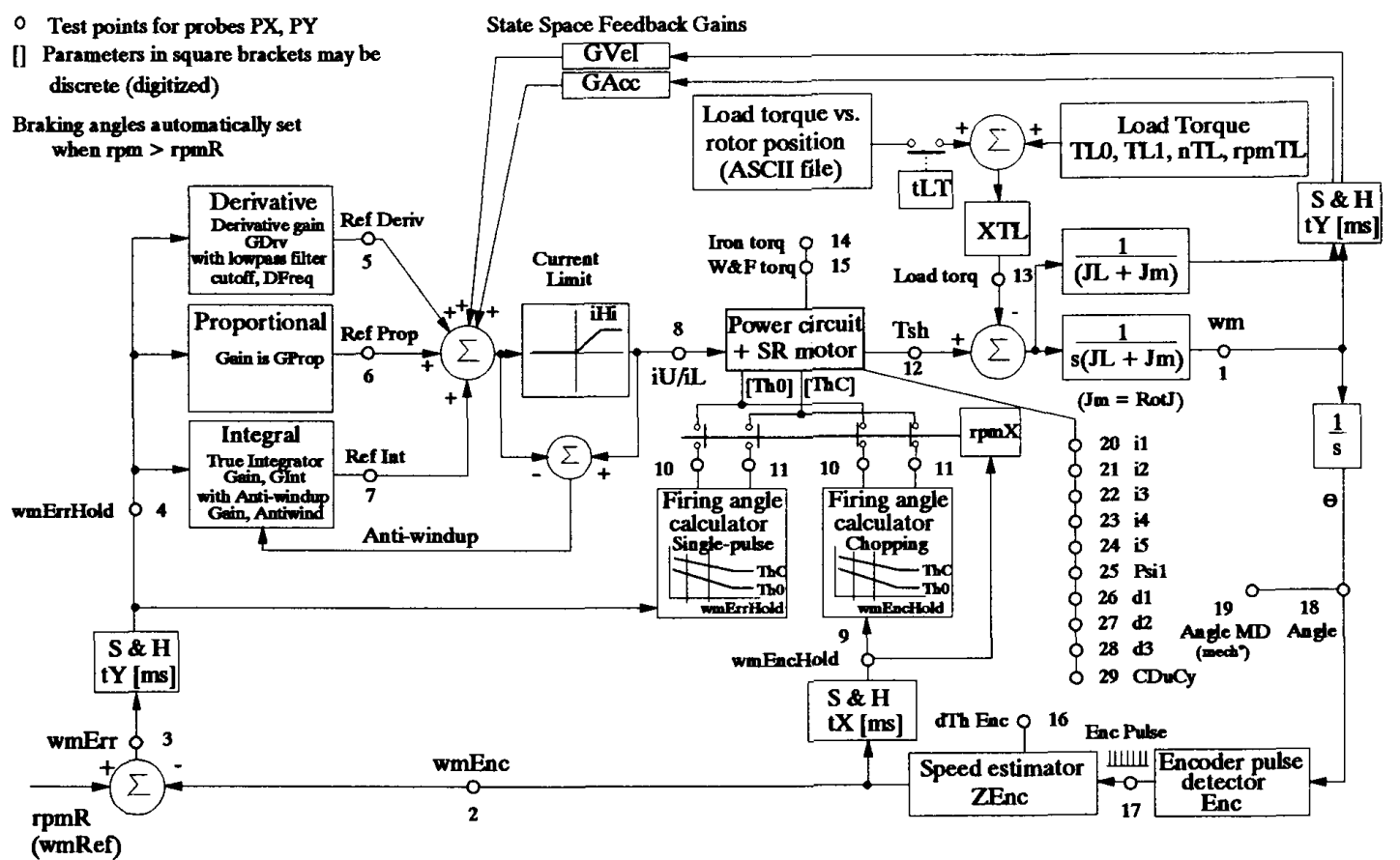

Fig. 10. Block diagram of $P C-S R D$ Dynamic computer program.

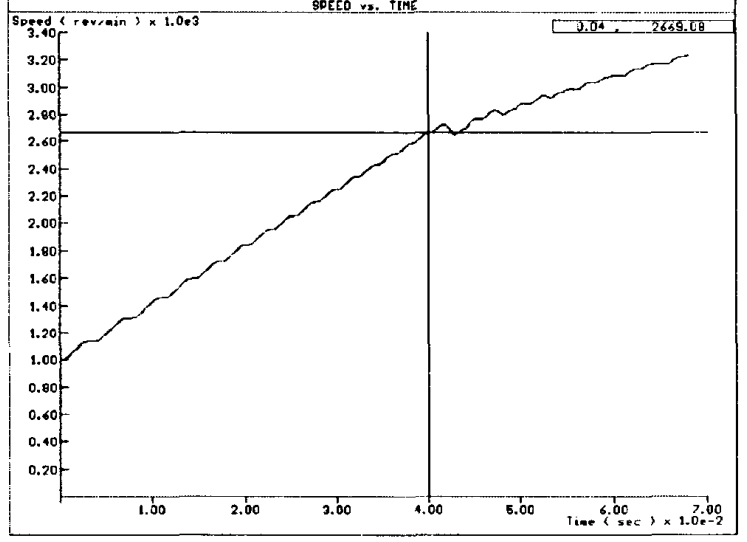

Fig. 11. Speed-versus-time transient in which a short circuit fault occurs at the terminals of phase 1 (just after the cursor position).

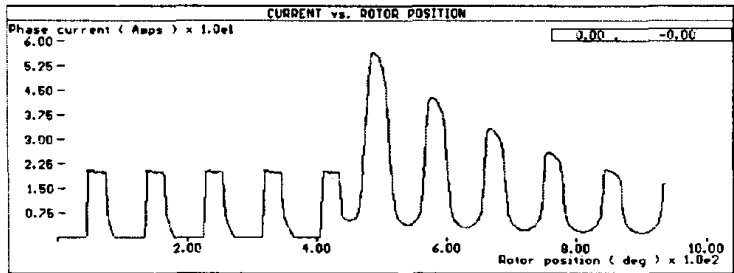

Fig. 12. Phase 1 current versus time during short-circuit fault on phase 1 .

which time the phase 1 current never reaches zero. Of course, the unbalanced forces discussed earlier will be excited by the peak current, which may be several times larger than the rated peak current.
The high current pulses continue to be generated for as long as there is stored energy remaining in the magnetic circuit of phase 1 . This energy is dissipated by the phase resistance (or what remains of it through the fault), and the current pulses gradually decay to zero over a period of many cycles. With no externally applied voltage, the pulses cannot be sustained indefinitely; however, energy can be converted from mechanical to electrical by phase 1 , increasing the heat dumped into the fault and depleting the net shaft torque. That this is so is evident from the fact that the post-fault acceleration is only $50 \%$ of the pre-fault value, not $2 / 3$. Fig. 13 is a reproduction of Fig. 12 with the other phase currents and the torque added to the graphs.

Fig. 14 shows the flux-linkage/current locus of phase 1 . Before the fault, the current is regulated normally at $20 \mathrm{~A}$, and several loops are traversed uniformly. The instant of the fault can clearly be seen on the diode-conduction section of the locus, and immediately the generating action drives the current up to a high value. As the flux decays, the current peaks become smaller.

So far it has been assumed that the fault occurred at the terminals of the winding, and the calculation in Figs. 11-14 employs the actual phase resistance. Similar consequences would follow a short-circuited turn. Also hazardous is the possibility of a short-circuit in certain parts of the controller itself. The current pulses are unidirectional and have no zerocrossings for a long time, and therefore a current waveform of this type could just as well flow through one of the freewheeling diodes and one of the phase transistors if the other phase transistor has failed to commutate sufficiently early to permit 


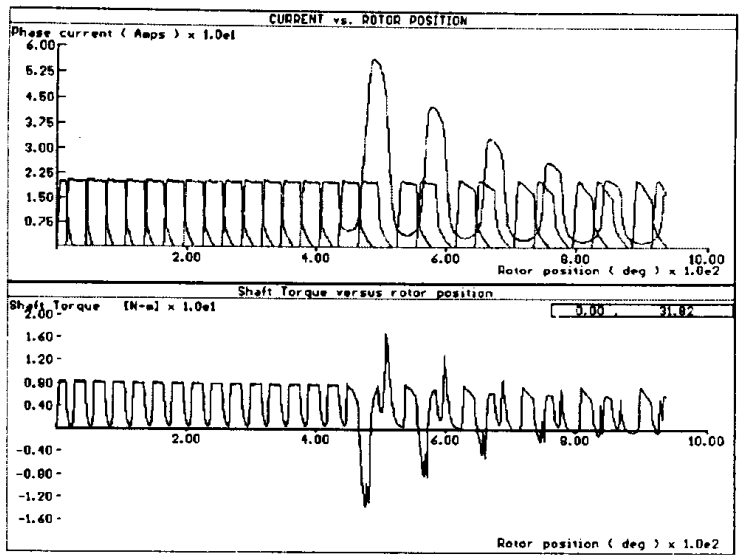

Fig. 13. All currents and torque versus time during short-circuit fault on phase 1.

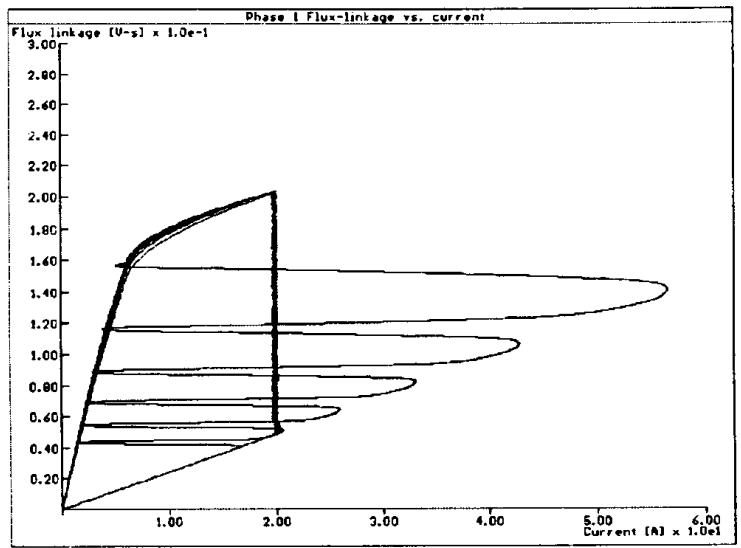

Fig. 14. Flux-linkage/current during short-circuit fault on phase 1.

the flux to decay to zero before the generating phase begins. In this event the conducting transistor would be expected to interrupt the fault current as soon as it reaches a preset limit. This implies that both transistors must be individually capable of current-limiting and of carrying transient currents much higher than normal.

\section{Winding Configuration and Protection}

Various winding configurations for the SRM are shown in Fig. 15, including series and parallel connection of opposite pole-coils and the use of separate phase-legs for increased redundancy. A summary of the main characteristics of these configurations is given below, because it is relevant in defining the problem of unbalanced forces and operation under fault conditions.

\section{A. Single Windings: Series Connection (Fig. 15):}

This is the most common arrangement. The currents in the two pole-coils are automatically equal under normal conditions. If there is magnetic asymmetry between the opposite
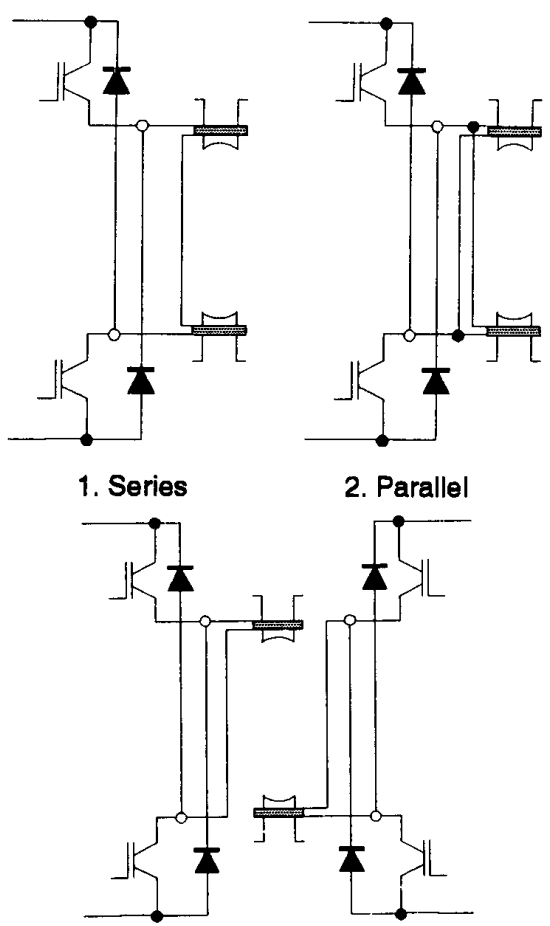

3. Independent

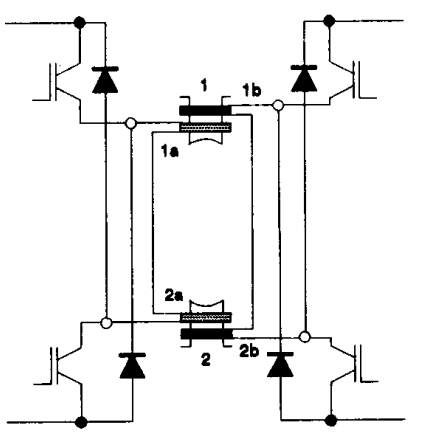

4. Double Series

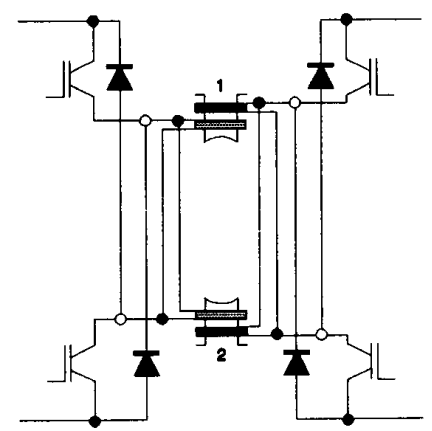

5. Double Parallel

Fig. 15. Complete set of winding/converter configurations.

poles, the flux-linkage waveforms differ, and the voltages and mechanical forces associated with the two poles differ; in other words, series connection per se does not guarantee balanced 
voltage-sharing. Therefore it does not guarantee balanced fluxlinkages or balanced forces.

If coil 1 becomes open-circuited, the phase current is zero. All torque and all forces associated with the phase are zero, and there is no possibility of electrical or magnetic interaction with other healthy phases. This condition can be detected by the current sensors that would ordinarily be included in the controller.

If coil 1 becomes short-circuited, even with a single shorted turn, its voltage will be zero and all the applied voltage will appear across coil 2 . In current-regulated operation (lowspeed motoring), the controller will automatically limit the current; however, the chopping frequency or current ripple may increase (by a factor of about 2), depending on the current-regulator strategy, and $d i / d t$ will be twice normal.

Much of the discussion of the "fault tolerance" of the SR machine has been based on the series connection (see Fig. 15) and the tacit assumption that faults will occur at the phase terminals rather than at the terminals of individual coils (or within individual coils). Within this restricted view, the SR machine obviously has a high level of fault tolerance, but in reality the picture is much more complicated.

\section{B. Single Windings: Parallel Connection of Pole Coils}

This arrangement is useful for high-speed machines because it permits the use of thinner conductors and more turns per pole, where the series connection would require thick conductors and a small number of turns.

If coil 1 becomes open-circuited, the applied voltage remains the same on coil 2. At low speeds the current-regulator will try to maintain normal phase current, so the current in coil 2 will double. In single-pulse operation (high-speed motoring and generating) the applied voltage pulse will cause the fluxlinkage waveform in coil 2 to have its normal value, but because of the magnetic unbalance, the current will not have its normal waveshape. The actual current waveshape is difficult to predict. The current-regulator may still be able to limit the total phase current to a preset value, but this will be twice the current normally allowed in one pole-coil. The unbalanced forces will cause serious noise and vibration.

If coil 1 becomes short-circuited, this is equivalent to a shoot-through fault in an ac inverter, and is potentially worse because the two transistors are normally gated simultaneously and the controller, acting normally, will try to keep them both on at the same time. Only a fast-acting current-regulator can protect the transistors against failure, by turning them off quickly. The di/dt under these conditions will be extremely high. While this fault persists, the full energy of the dc filter capacitors is liable to be dissipated in the fault and the transistors.

\section{Single Windings: Independent Drives}

In this arrangement, each pole-coil has its own independent half-bridge controller. These controllers can remain isolated from each other only if there are two separate dc systems.

If coil 1 becomes open-circuited, no current flows. Coil 2 is electrically independent and continues to receive voltage

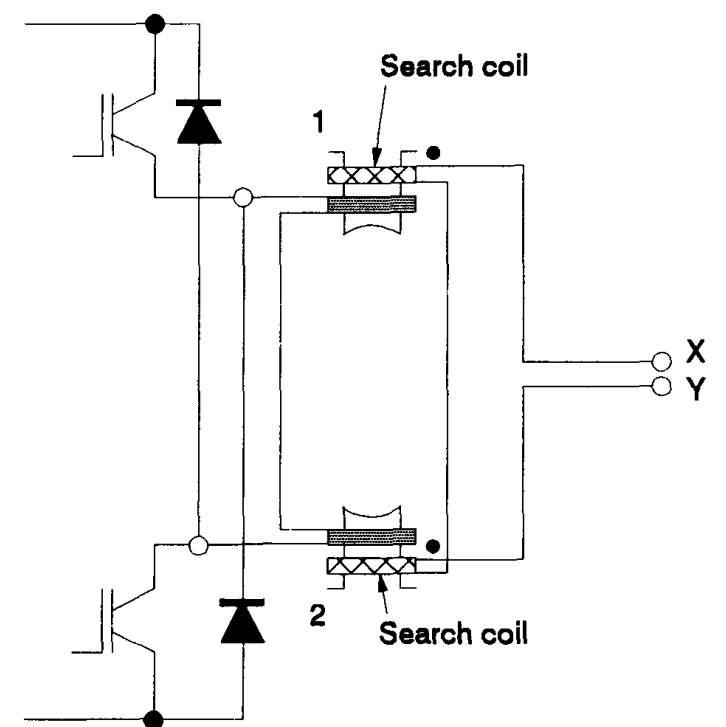

Fig. 16. Differential voltage sensing with search coils proposed by C. M. Stephens [2].

pulses, but its current waveform will be abnormal because of the loss of magnetic balance. There will be significant mechanical unbalance with vibration.

If coil 1 becomes short-circuited, the "shoot-through" fault arises as in $B$. However, the circuit of coil 2 is not directly affected.

Generally, in high-speed SR machines coils 1 and 2 will be on diametrically opposite poles. This minimizes the risk of consequent damage to coil 2 , if coil 1 fails catastrophically. However, other phases may have coilsides in the same slots as coil 1 , and they may be vulnerable to damage.

This arrangement appears to offer greater security than systems 1 or 2 provided that the two controllers are truly isolated from each other, and provided that the magnetic and mechanical unbalance is tolerable.

\section{Double Windings: Series Connection}

This arrangement has the same advantages as (Section V-C), but eliminates the magnetic and mechanical unbalance when one section is faulted. The fault characteristics of each section are similar to those of (Section V-A).

\section{E. Double Windings: Parallel Connection}

This arrangement also has the same advantages as (Section V-C), and it eliminates the magnetic and mechanical unbalance when one section is faulted. The fault characteristics of each section are similar to those of (Section V-B).

Figs. 16 and 17 show two protective schemes capable of detecting unbalanced faults in opposite pole-coils.

\section{F. Differential Voltage Sensing with Search Coils (Fig. 16)}

This scheme, proposed by Stephens [2], is shown for the single-winding, series connection. However, it can be adapted for use with any of the arrangements shown in Fig. 15. 


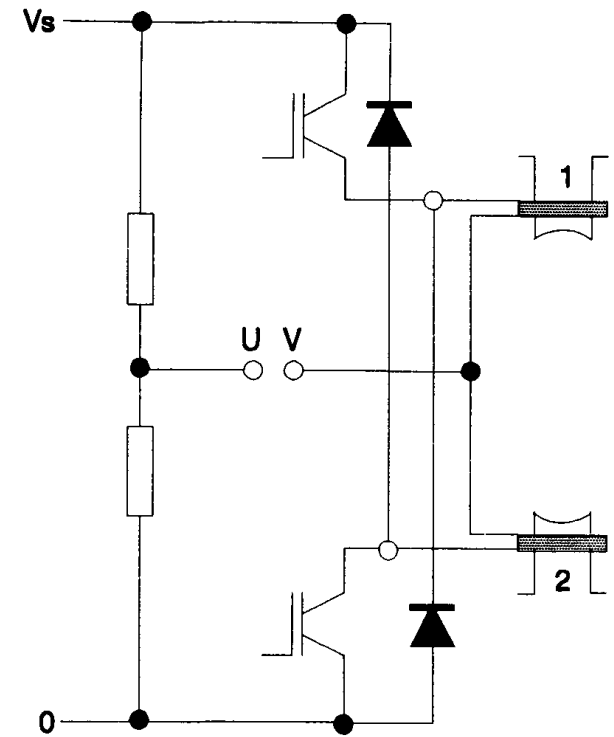

Fig. 17. Differential voltage sensing without search coils.

Under normal operation the flux-linkage waveforms in coils 1 and 2 are identical, and therefore the induced EMF's in the search coils are equal and opposite. If they are connected in opposition, the normal voltage $X Y$ is zero. Any voltage appearing at terminals $X Y$ indicates unbalance between the coils. This arrangement is also capable of detecting unbalance caused by mechanical asymmetry.

\section{G. Differential Voltage Sensing with No Search Coils (Fig. 17)}

This is shown for the single-winding, series connection. However, it can be adapted for use with any of the arrangements discussed in Section V. Functionally the scheme is the same as before, but the main coils are themselves used as the search-coils. Under normal operation the potential at terminal $V$ is half the supply voltage (i.e., $V_{s} / 2$ ). Unbalance is detected by comparing the potential at terminal $V$ with the potential at a reference terminal $U$, which is obtained from a voltage divider or potentiometer.

An advantage of this arrangement is that it eliminates the need for separate search coils, and requires only one additional terminal instead of two. However, bringing terminal $V$ out to an external comparator circuit may itself compromise the reliability of the system.

The system operates only when there is a conduction path through both transistors or both diodes, and it requires that the voltage drop is the same in corresponding devices. In some modes of operation (e.g., low-speed motoring), it is normal to operate the SR machine with one transistor chopping while the other one is held on for a whole conduction period. In this case the voltage at terminal $V$ deviates from $V_{s} / 2$ by the difference between the transistor volt-drop and the diode voltdrop, which may be of the order of $2 \mathrm{~V}$ in a $270 \mathrm{~V}$ system. This sets a limit on the sensitivity of the detection system. During high-speed motoring or generating, it is normal to operate both transistors identically, and the voltage deviation at terminal $V$ is then reduced to the difference in the volt-drops across the two transistors (or the two diodes) while they are carrying the same current.

\section{DISCUSSION AND CONCLUSION}

The "fault tolerance" of the SR machine is not absolute, and must be viewed with several qualifications. Three particular hazards that can arise under fault conditions have been identified and analyzed in this paper:

1. Unbalanced lateral forces on the rotor caused by electrical faults;

2. Unbalanced forces cause by mechanical offset of the rotor; and

3. Uncontrollable pulsed currents in a shorted coil.

A simplified magnetic analysis suggests that electrical faults on single coils produce higher lateral forces than those due to mechanical offset of the rotor, and that these forces and their waveforms can be estimated by simple order-of-magnitude methods. Finite-element analysis and measurements have confirmed the simplified analysis in general terms, but the present paper attempts no more than a superficial analysis and much more work is needed in this area.

The prediction of transient fault currents in the motor and controller is a more complex problem requiring the use of specialized simulation software such as the PC-SRD Dynamic program. Simulations with this program show the possibility of large pulsed currents in a shorted phase winding when the fault occurs during a period of diode conduction. Such faults occurring within the machine cannot necessarily be isolated by switching actions in the controller. The paper discusses the relative merits of different winding configurations to minimize the impact of this type of fault. Protective schemes employing various forms of differential voltage sensing (as originally proposed by Stephens) can isolate the controller from faulty circuits in the machine, and these are discussed in order to set the scene for a more thorough study of the unbalanced fault problem.

This future work needs to concentrate on the analysis and measurement of unbalanced forces, and on further investigation of the dynamic performance of the SR machine under abnormal conditions. Results achieved in this paper indicate that a combination of analysis tools is required, including measurement, simulation software, finite-element analysis, and traditional engineering analysis and approximation.

\section{ACKNOWLEDGMENT}

The author would like to thank J. Kelly, P. Miller, and R. P. Deodhar for assistance with measurements, Dr. G. Gray for his contribution to PC-SRD Dynamic, and the companies of the SPEED Consortium. Thanks are also due to Leon Kasdan and Dick Chu (Allied Signal) for helpful discussions in connection with Section $\mathrm{V}$. The finite-element calculations were performed using PC-OPERA from Vector Fields Ltd.

\section{REFERENCES}

[1] T. J. E. Miller, Switched Reluctance Motors and Their Control. Published jointly by Hillsboro, OH: Magna Physics Tridelta and London, UK: Oxford University Press, 1993. 
[2] C. M. Stephens, "Fault detection and management system for fault tolerant switched reluctance motor drives," IAS Annu. Meeting, pp. 574-578, 1989.

[3] T. J. E. Miller and M. McGilp, "Nonlinear theory of the switched reluctance motor for rapid computer-aided design," in IEE Proc., vol. 137, pt. B, no. 6, pp. 337-347, Nov. 1990.

[4] _ "High-speed CAD for brushless motor drives," European Power Electron. Conf., Florence, Italy, pp. 3.435-3.439, Sept. 3-6, 1991.

[5] D. A. Staton, M. I. McGilp, T. J. E. Miller, and G. Gray, "High-speed PC-based CAD for motor drives," European Power Electron. Conf., Brighton, UK, Sept. 13-15, 1993, pp. 6.26-6.311.

[6] W. F. Ray, R. M. Davis, and R. J. Blake, "The control of SR motors," Conf. on Appl. Motion Contr., Minneapolis, MN, June 1986, pp. $137-145$.

[7] K. Takayama et al., "Thrust force distribution on the surface of stator and rotor poles of switched reluctance motor," IEEE Trans. Magn., vol. 25, no. 5, pp. 3997-3999, Sept. 1989.

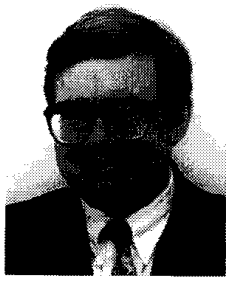

Timothy J. E. Miller (M'74-SM'82) is a native of Wigan, UK. He was educated at Atlantic College and the University of Glasgow, Scotland, and the University of Leeds.

From 1979 to 1986 he was an electrical enginee and program manager at General Electric Corporate Research and Development, Schenectady, NY. His industrial experience includes periods with GEC (UK), British Gas, International Research and Development, and a student-apprenticeship with Tube Investments Ltd. He is Lucas Professor in Power Electronics, and founder and Director of the SPEED Consortium at the University of Glasgow, Scotland. He is the author of 105 publications in the fields of motors, drives, power systems and power electronics.

Prof. Miller is a Fellow of the Royal Society of Edinburgh and a Fellow of the IEE. 